\title{
Fuzzy logic control of a nitrogen laser
}

\author{
Siu-Chung Tam, MEMBER SPIE \\ Siong-Chai Tan \\ Wah-Peng Neo \\ Sze-Chern Foong \\ Choon-Hao Chan \\ Anthony T. S. Ho \\ Hock-Chuan Chua \\ Nanyang Technological University \\ School of Electrical and Electronic \\ Engineering \\ Division of Microelectronics \\ Photonics Research Group \\ Nanyang Avenue \\ Singapore 639798 \\ E-mail: esctam@ntu.edu.sg

\section{Sing Lee} \\ Nanyang Technological University \\ National Institute of Education \\ School of Science \\ 469 Bukit Timah Road \\ Singapore 259756
}

\begin{abstract}
Traditionally, the stability of the output of a laser is controlled through scientific means or by a simple feedback loop. For multiinput multioutput control and for medium- to high-power lasers, however, these control schemes may break down. We report on the use of a fuzzy logic control scheme to improve the stability of a pulsed nitrogen laser. Specifically, the nitrogen laser is modeled as a two-input two-output system. The two input parameters are the discharge voltage $(V)$ and nitrogen pressure $(P)$, and the two output parameters are the pulse energy $(E)$ and pulse width (PW). The performance of the fuzzy logic controller is compared with a decoupled two-channel PID (proportional+integral + derivative) controller. In our experiment, the long-term stabilities of the open-loop system are $1.82 \%$ root mean square (rms) for pulse energy and $4.58 \%$ rms for pulse width. The PID controller achieves better performance with long-term stabilities of $1.46 \%$ rms for pulse energy and $4.46 \%$ rms for pulse width. The fuzzy logic controller performs the best with long-term stabilities of $1.02 \%$ rms for pulse energy and $4.24 \% \mathrm{rms}$ for pulse width, respectively. (c) 2001 Society of Photo-Optical Instrumentation Engineers. [DOI: 10.1117/1.1336530]
\end{abstract}

Subject terms: lasers; nitrogen lasers; fuzzy logic; laser stability; fuzzy control.

Paper 990263 received July 6, 1999; revised manuscript received May 23, 2000; accepted for publication Aug. 29, 2000.

\section{Introduction}

It is important to control the stability of the output characteristics of any laser. For example, in excimer laser keratectomy, the control of the laser duration is essential in determining the amount of ablation of the cornea tissues. Similarly, the temporal characteristics of the laser pulse are vital in applications such as shadowgraphy, schlieren photography, microlithography, and micromachining.

Generally speaking, there are two ways to control the stability of the output beam of a laser system. The first approach uses scientific means to examine the properties of the system and compensate the errors by redesigning or modifying system parameters analytically. The second approach recognizes the difficulty in deriving a mathematical formula to relate all the parameters causing the variations, and introduces engineering control techniques such as the conventional PID (proportional+integral+derivative) control, expert systems, and computational intelligence to achieve system stability. The PID technique is simple to implement but does not work well for multiinput multioutput (MIMO) systems. Moreover, the system parameters must be fine-tuned for different set points. Expert systems are heuristic in nature and require extensive adjustments for quantitative control. Computational intelligence-which encompasses artificial neural networks, fuzzy logic, neurofuzzy, fuzzy-neural, and genetic algorithms - are very promising candidates in achieving stable control of MIMO systems ${ }^{1-5}$.

In this paper, we report on the results of implementing a fuzzy logic controller on an experimental nitrogen laser. The performance of the fuzzy logic controller is compared with that obtained for free-running operation and that obtained with a two-channel PID controller.

\section{Experimental Setup}

The discharge circuit for the experimental laser is of the conventional Blumlein design modified by the on-demand pulse charging technique developed by Lee et al. ${ }^{6}$ The discharge voltage operates from 9.0 to $11.1 \mathrm{kV}$, and the nitrogen pressure can be varied from 250 mbar to 1.0 bar. For convenience of 8-bit numerical control from a PC, each of the discharge voltage $(V)$ and nitrogen pressure $(P)$ is normalized to a range of 0 to 255 , respectively. A schematic diagram showing the experimental setup is shown in Fig. 1.

The low-inductance parallel plate capacitors $\mathrm{C} 1$ and $\mathrm{C} 2$ are charged by the high-voltage circuit. The trigger delay is used to set the discharge voltage at the desired level within the range of 9.0 to $11.1 \mathrm{kV}$. The pressure of the nitrogen gas that flows into the laser head is controlled by a SMC IT209-202 electrovacuum regulator.

The output beam from the $\mathrm{N}_{2}$ laser is split into two paths for simultaneous measurements of pulse energy and pulse width. The pulse energy is measured with a Molectron J-25 pyroelectric detector connected to a Molectron EM500 energy meter.

The digital signal bearing the energy content of the pulse is sent to the PC controller via a RS232 link. The pulse width is measured with a MG 13DAH 001 photodiode. The signal is relayed to and displayed on an HP 54270D realtime oscilloscope that has a sampling rate of 8 gigasamples/ second $(\mathrm{GSa} / \mathrm{s})$. The oscilloscope is linked to the PC controller via a general purpose interface bus (GPIB) interface. $^{7}$ 


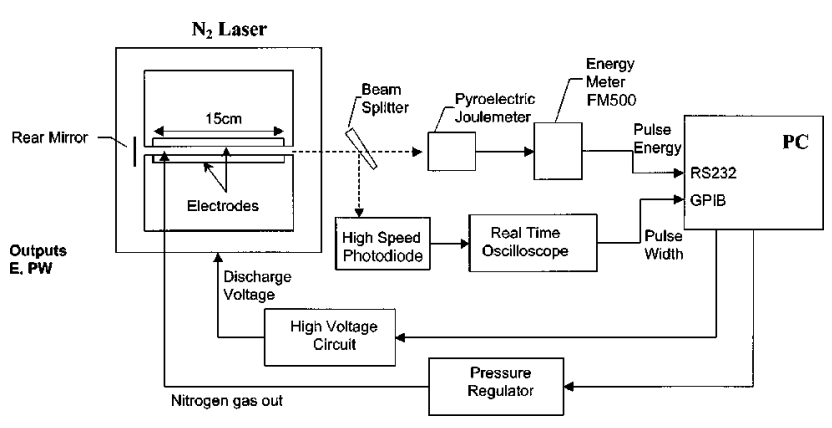

Fig. 1 Schematic of the control loop.

The fuzzy logic controller is implemented using the MATLAB ${ }^{\circledR}$ Fuzzy Logic Toolbox executed from the PC. The controller adjusts the discharge voltage $(V)$ and nitrogen pressure $(P)$ by monitoring the actual energy output $(E)$ and pulse width (PW) to achieve set-point equilibrium. An interface card was built for this purpose, although any commercial general-purpose I/O card can be used.

\section{Fuzzy Logic Controller}

Fuzzy logic is a computational technique that enables the solving of model-based design problems using both intuition and heuristics. It enables the representation of system behavior without the need for vigorous mathematical system modeling. Figure 2 shows a block diagram of the fuzzy logic controller that was implemented for the nitrogen laser.

The desired targets for pulse energy $\left(E_{0}\right)$ and pulse width $\left(\mathrm{PW}_{0}\right)$, were input to the fuzzy logic controller. The controller generates digitized outputs to control the discharge voltage $(V)$ and nitrogen pressure $(P)$, which are used to operate the nitrogen laser.

Two membership functions were employed in the fuzzy logic controller. The input membership function was formulated based on the error signal expressed as a percentage of the desired signal and was used to fuzzify the measured pulse energy and pulse width into suitable linguistic variables. The output membership function was modeled on the fuzzy control (if-then) rules, and output crisp adjustments in voltage $(\Delta V)$ and pressure $(\Delta P)$ that stabilize the laser pulse energy and pulse width simultaneously. A detailed discussion of the implementation of the fuzzy logic controller is provided in Sec. 5.

The fuzzy if-then rules were formulated by recording the field data collected by firing the laser at different voltage and pressure settings. Ten readings were collected for each combination of voltage and pressure setting, and the average values are plotted in Figs. 3 and 4, respectively. We

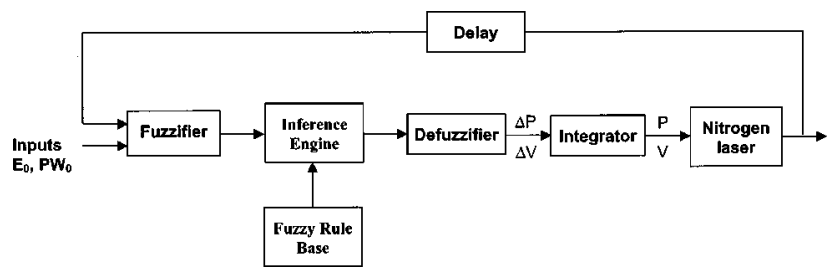

Fig. 2 Fuzzy logic controller.

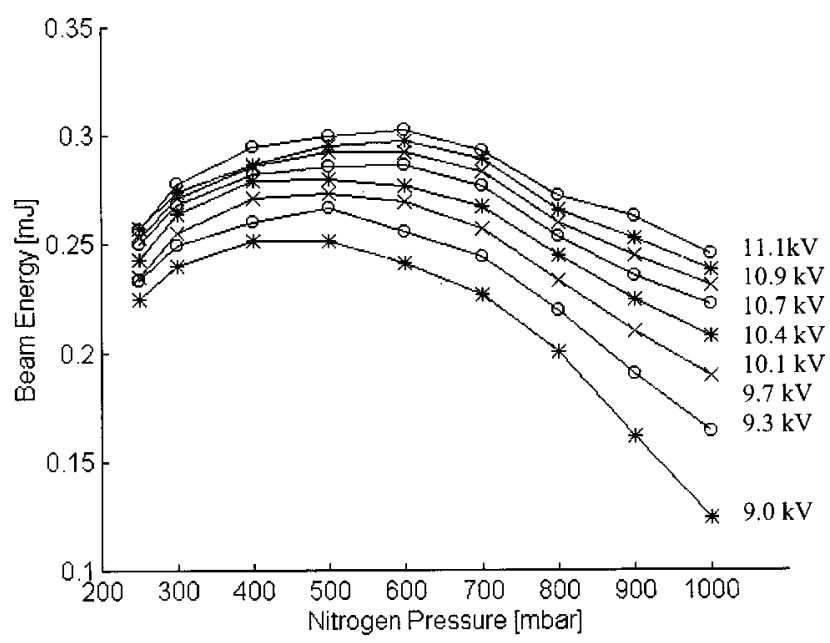

Fig. 3 Pulse energy versus pressure for different discharge voltages.

observe that the voltage has a higher impact on the output energy, while pressure has a higher impact on the pulse width.

Interpreting the fuzzy if-then rules involves distinct parts: first evaluating the antecedent (which involves fuzzifying the input and applying fuzzy operators), and second applying that result to the consequence (known as implication). The consequence specifies a fuzzy set to be assigned to the output. The implication function then modifies that fuzzy set to the degree specified by the antecedent.

Finally, the outputs, steps change in voltage $(\Delta V)$ and pressure $(\Delta P)$ after defuzzification, are obtained. These changes are added to the $(V, P)$ settings used in the previous firing sequence. Defuzzification is defined as a mapping from a space of fuzzy control actions to a space of crisp control action. The defuzzification is based on the center-of-area (COA) method and adjusts the voltage and pressure settings for the next firing instance. The defuzzification based on COA is given by

$Z_{\mathrm{COA}}=\frac{\int_{z} \mu_{c^{\prime}}(z) z \mathrm{~d} z}{\int_{z} \mu_{c^{\prime}}(z) \mathrm{d} z}$,

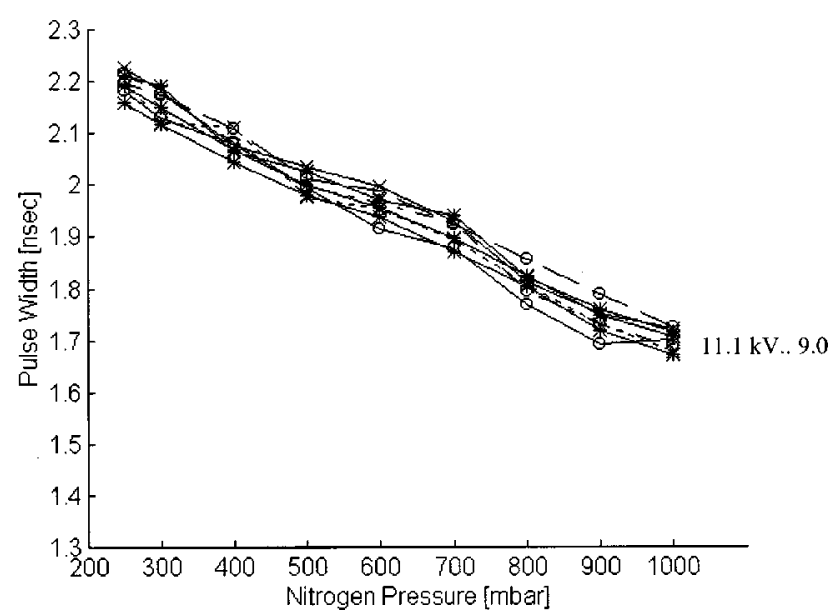

Fig. 4 Pulse width versus pressure for different discharge voltages. 


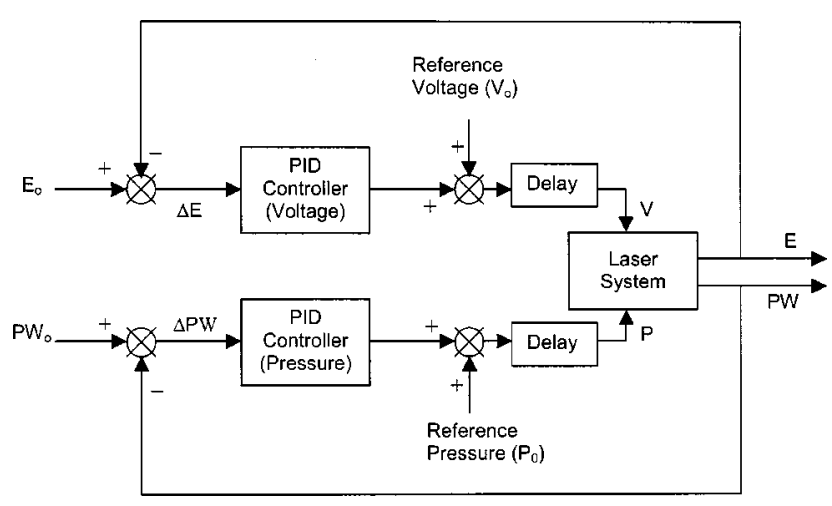

Fig. 5 Block diagram of two-channel PID controller.

where $\mu_{c^{\prime}}(z)$ is the membership function of the consequence after implication. In this work, the Mandami implication method was used. ${ }^{3}$

\section{Implementation of a PID Controller}

The PID controller is a well-established control scheme. It has also been implemented to benchmark the performance of the fuzzy logic controller. The discrete-time PID model is given by

$$
\begin{aligned}
u_{k}= & K_{p} e_{k}+\frac{1}{2} K_{i} \Delta T\left[e_{0}+2 e_{1}+2 e_{2}+\cdots+2 e_{k-2}+e_{k-1}\right] \\
& +\frac{K_{d}}{\Delta T}\left(e_{k}-e_{k-1}\right)
\end{aligned}
$$

where $u_{k}$ is the control action at the $k$ 'th step, $e_{k}$ is the error between the actual output and the desired output at the $k$ 'th step, and $\Delta T$ is the time interval. We can assume that $\Delta T$ $=1$, as its numerical value will not affect the actual system performance. The three terms $K_{p}, K_{i}$, and $K_{d}$ determine the proportional gain, steady state error, and the response time to a fast changing input, respectively.

Two versions of the PID control algorithm were developed. In the first one, we assumed that there is no correlation between discharge voltage and pulse width, and between gas pressure and pulse energy. This assumption led to an independent two-channel two-variable PID control scheme. The block diagram for this configuration is shown in Fig. 5. The values of $K_{p}, K_{i}$, and $K_{d}$ are derived approximately from Figs. 3 and 4 at the settings of $E_{0}$ $=0.25 \mathrm{~mJ}$ and $\mathrm{PW}_{0}=1.90 \mathrm{~ns}$.

In the second PID scheme, the correlations between the two input and output variables were taken into consideration. A coupled PID scheme was developed. ${ }^{4}$ However, we did not obtain any conclusive results as steady state stability cannot be reached for different sets of operating parameters.

\section{Implementation of a Fuzzy Logic Controller}

The fuzzy logic controller was implemented using the MATLAB toolkit. The use of MATLAB toolkit facilitates experimentation using different membership functions in the control scheme, as the toolkit includes many types of built-in membership, functions that include the triangular function; the trapezoidal function; the Gaussian curve, the bell function; the sigmoidal function; and the $Z, S$, and pi functions. As this is an experimental project that seeks to compare the practical effectiveness of using fuzzy logic in laser control, the MATLAB toolkit provides the flexibility and the ease of trying out different control schemes. Furthermore, the use of a standard software for parameter optimization is not restrictive, as MATLAB enables the generation of C-codes, so that a well-chosen fuzzy control algorithm can be implemented as a stand-alone executable program.

In general, fuzzy logic control could provide robust nonlinear control over the performance of a system through the selection of various input and output membership functions and the implementation of the fuzzy if-then rules. Usually, a composite three-class or a five-class membership function could yield reasonably good results in terms of correction range and system stability. In this project, the following five fuzzy logic (FL) control schemes were attempted:

1. Scheme FL1: a Z-function, three triangular functions, and an $S$ function are used for both the energy control and pulse width control.

2. Scheme FL2: this is similar to scheme FL1, but with slight variations in the output membership functions.

3. Scheme FL3: this is similar to scheme FL1, but with different output membership functions.

4. Scheme FL4: a $Z$ function, two Gaussian functions, a triangular function, and a $S$ function are employed.

5. Scheme FL5: this is similar to scheme FL3, but with a different range for the input variable energy_err.

The input membership functions for the various schemes are shown in Fig. 6, while the output membership functions are illustrated in Fig. 7. In these diagrams, the energy and pulse width error signals are represented by 'energy_err', and "pulse_err,' respectively. The compensation output signals are given by "delvoltage" and "delpressure," respectively.

Figure 8 shows the surface plots generated. The shape of the plots illustrates the incremented variations in the two output variables (delvoltage, delpressure) versus the variations in the two input variables (energy_err and pulse_err). Note that the surface plots correspond to the trends shown in Figs. 3 and 4. Thus, the use of different membership functions would enable a user to tailor the response graphs to the characteristic response of the system. Furthermore, the fuzzy logic controller allows for corrections in nonlinearities that are due to transducer response, measurement errors, environmental variations, etc. Using the preceding observations, the following 10 fuzzy if-then rules are formulated:

1. If (energy_err is "negative large"), then (delvoltage is "decrease more").

2. If (energy_err is "negative small"), then (delvoltage is "decrease little").

3. If (energy_err is "no error"), then (delvoltage is "no change").

4. If (energy_err is "positive small"), then (delvoltage is "increase little"). 

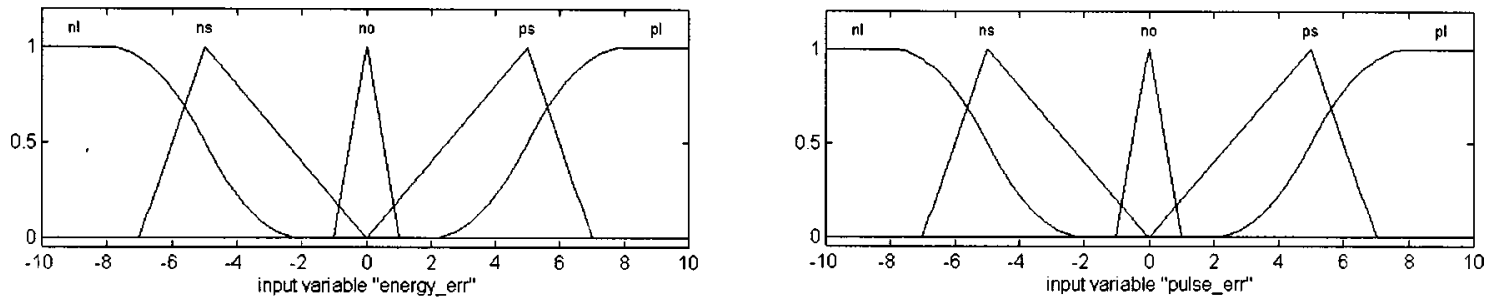

(a) Scheme FL1
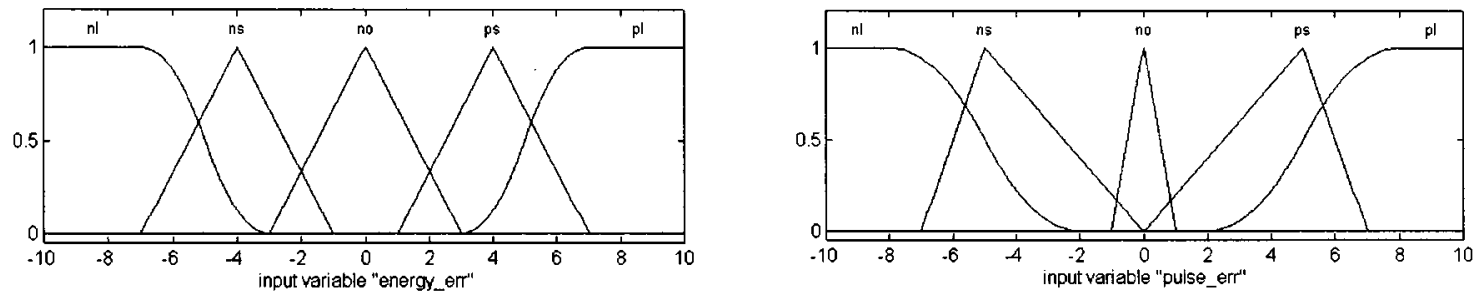

(b) Scheme FL2
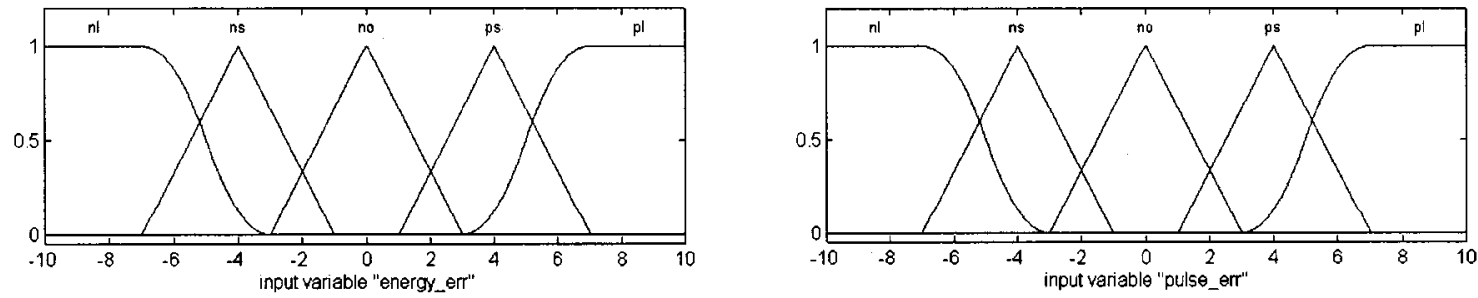

(c) Scheme FL3
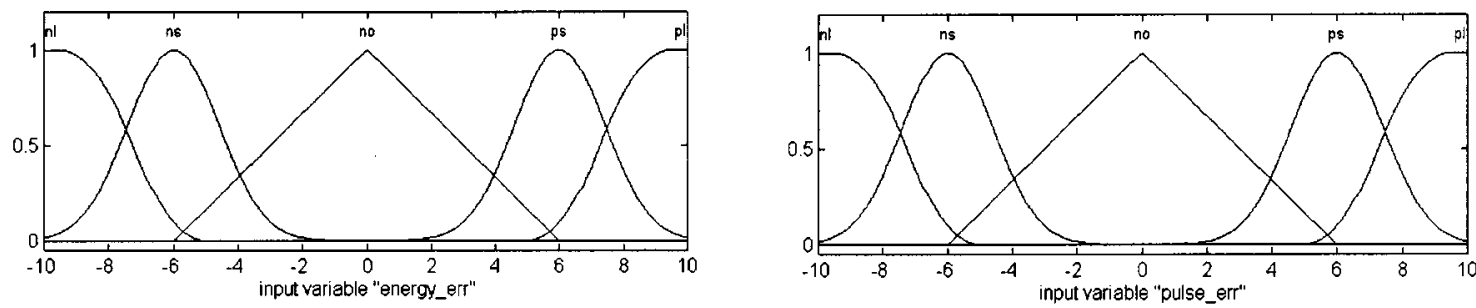

(d) Scheme FL4
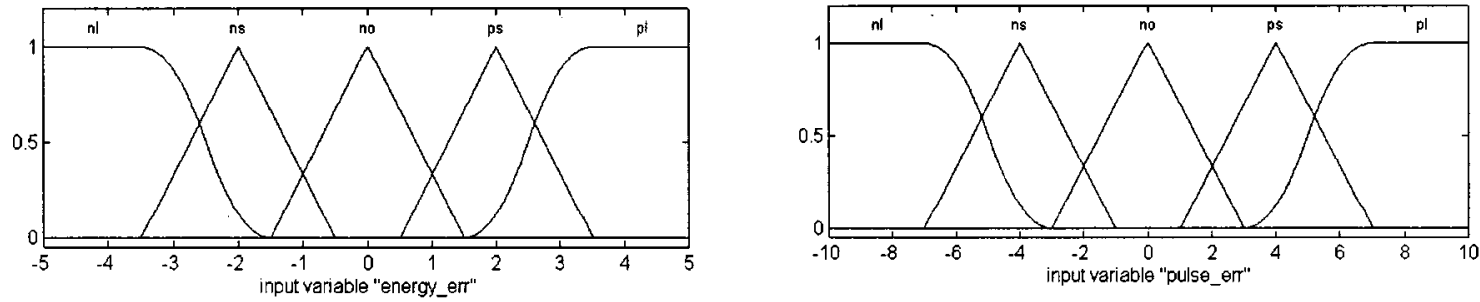

(e) Scheme FL5

Fig. 6 Input membership functions for various schemes.

5. If (energy_err is "positive large"), then (delvoltage is "increase more").

6. If (pulse_err is "negative large"), then (delpressure is "decrease more").

7. If (pulse_err is "negative small"), then (delpressure is "decrease little").

8. If (pulse_err is " "no error'), then (delpressure is " no change',).
9. If (pulse_err is "positive small"), then (delpressure is "increase little").

10. If (pulse_err is "positive large") then (delpressure is "increase more").

The five fuzzy logic control schemes were tested for their practical performance. From the experimental results obtained, it was found that scheme FL5 gave rise to the best 

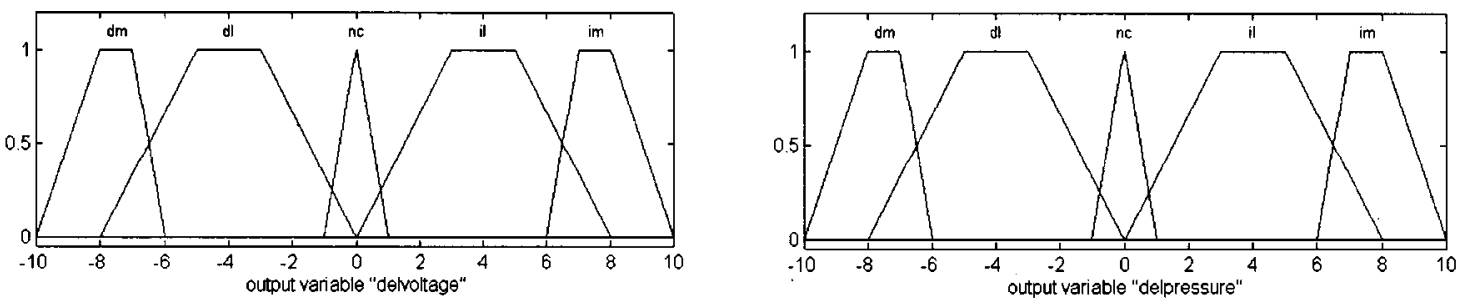

(a) Scheme FL1
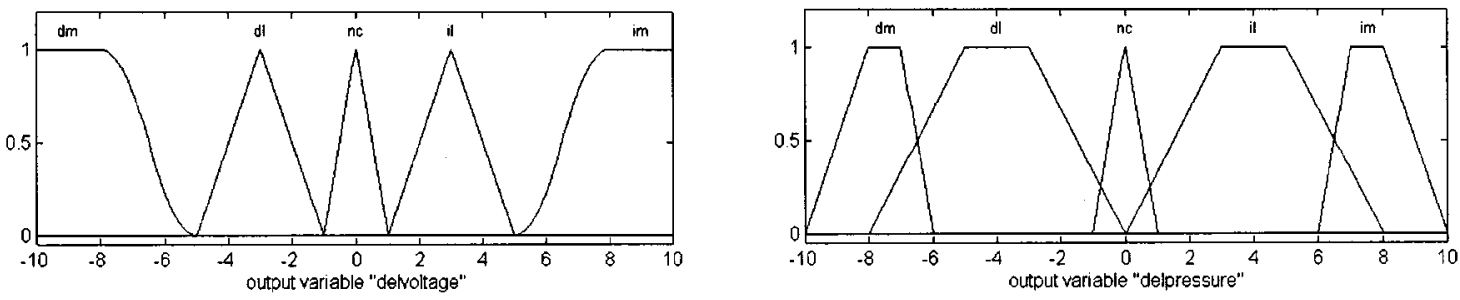

(b) Scheme FL2
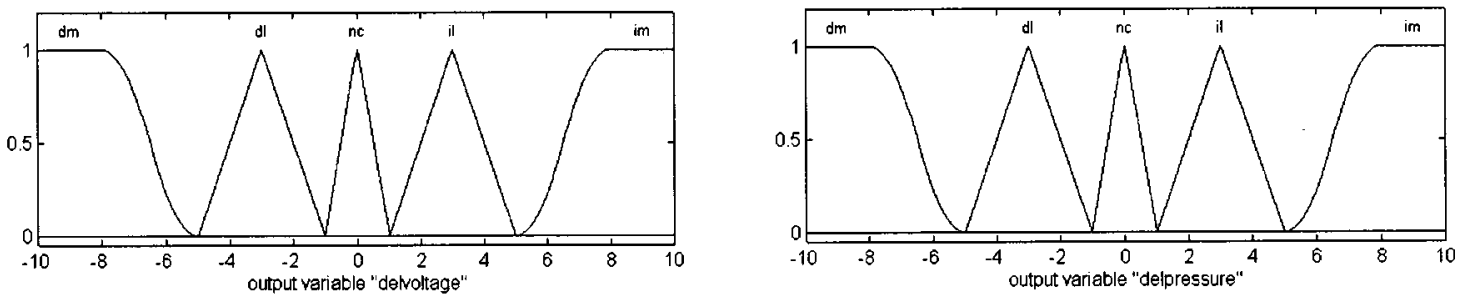

(c) Scheme FL3
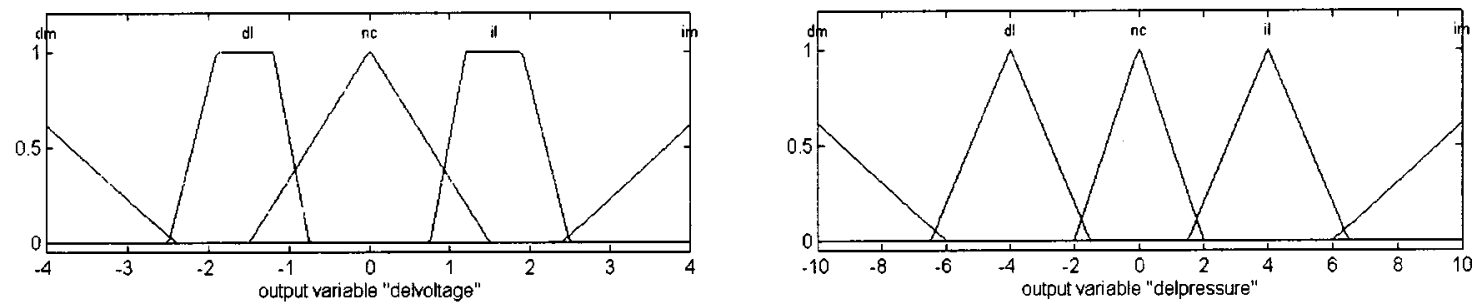

(d) Scheme FL4
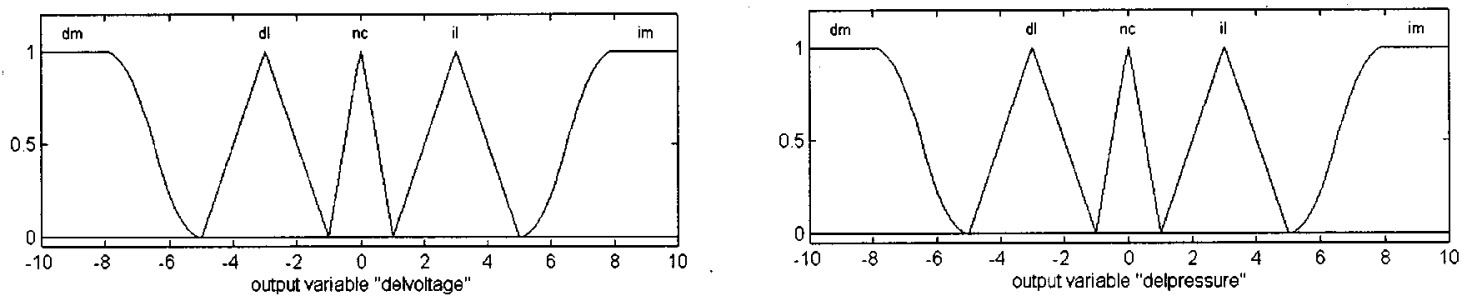

(e) Scheme FL5

Fig. 7 Output membership functions for various schemes.

performance. Hence scheme FL5 was implemented as the fuzzy logic controller for further comparisons.

\section{Results and Discussion}

The open-loop response of the nitrogen laser was used as the baseline reference. The temporal response for 4000 laser pulses, at a normalized discharge voltage of 220 and a normalized nitrogen pressure of 130 , and at a firing rate of 5 s/pulse, was recorded, as shown in Fig. 9. The average pulse energy and pulse width are $0.25 \mathrm{~mJ}$ and $1.97 \mathrm{~ns}$, respectively. The root mean squared (rms) instability for the pulse energy $(E)$ is $1.82 \%$, and that for the pulse width (PW) is $4.58 \%$. By taking the moving average of 20 consecutive pulses over the 4000 pulses recorded, the longterm drift was determined. It was found that the pulse energy drifts by $6.97 \%$ and the pulse width drifts by $6.63 \%$. The equations used for these calculations are

accuracy $=\left(1-\frac{|\overline{\bar{x}}-T|}{T}\right) \times 100 \%$, 

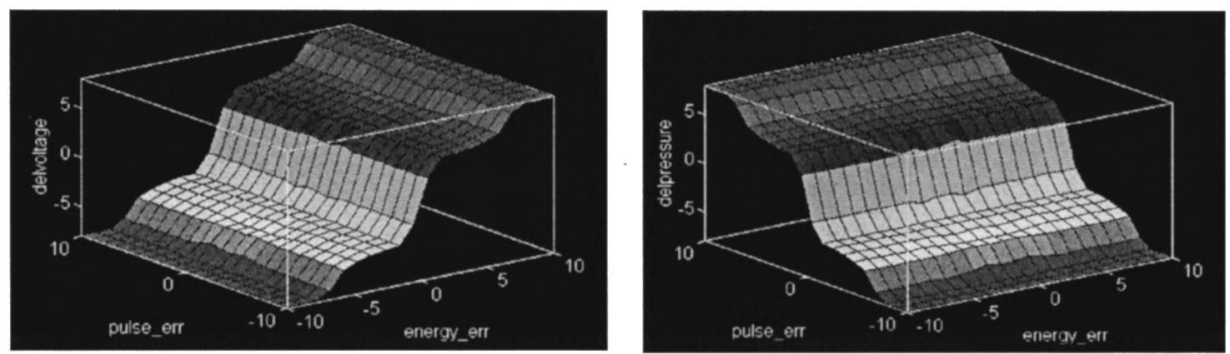

(a) Scheme FL1
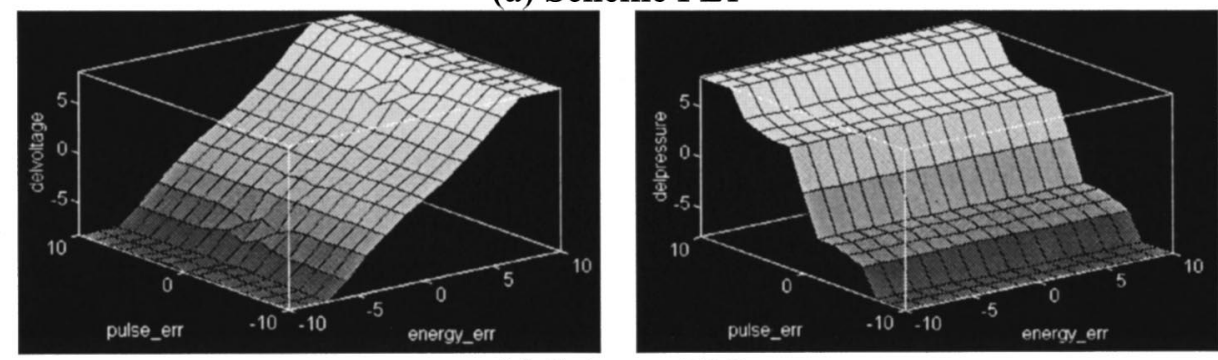

(b) Scheme FL2
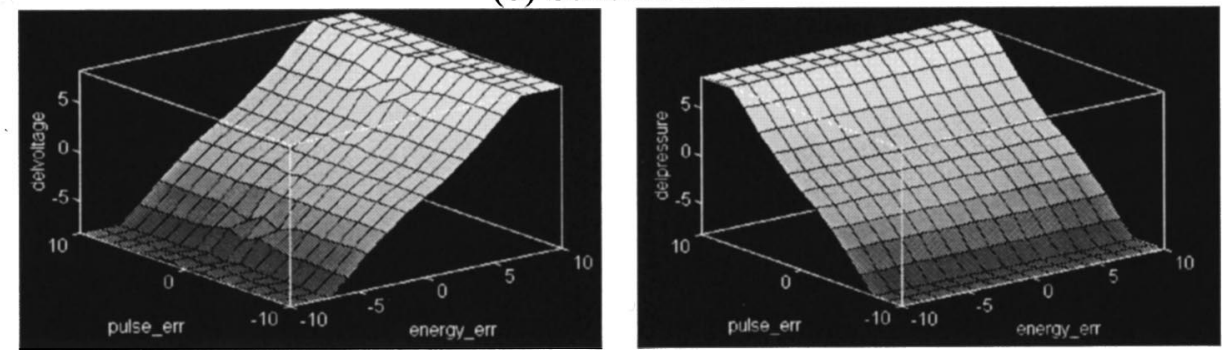

(c) Scheme FL3
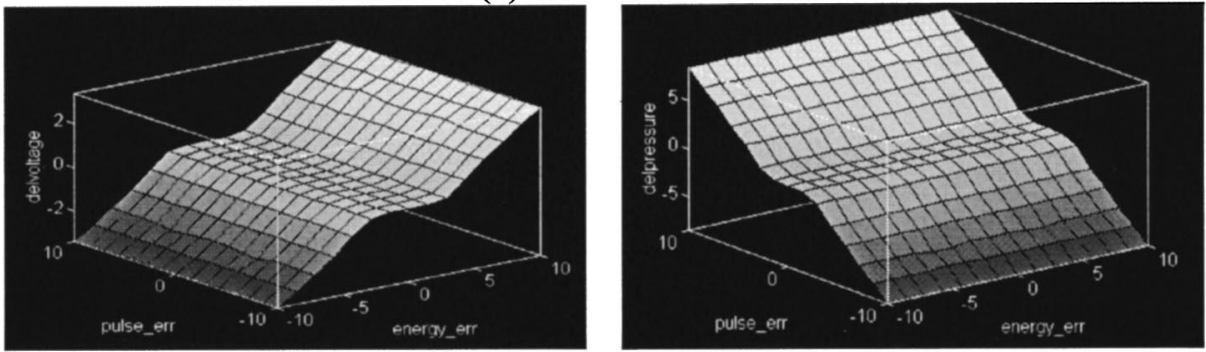

(d) Scheme FL4
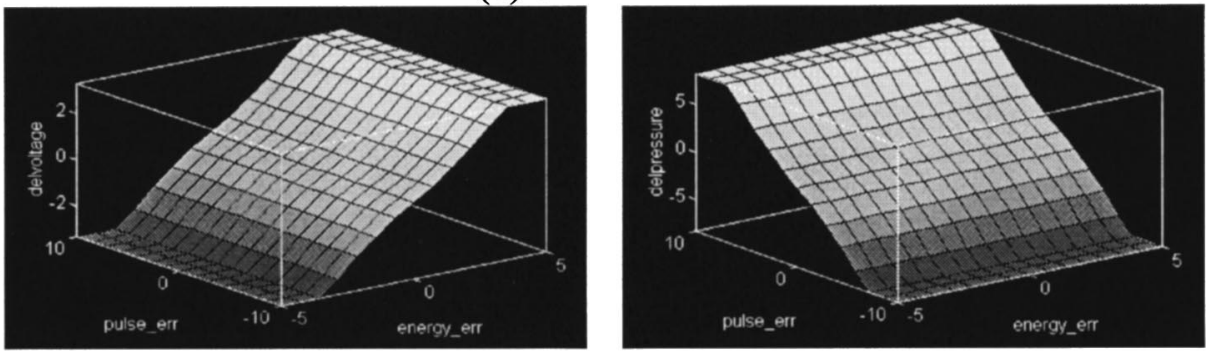

(e) Scheme FL5

Fig. 8 Surface plots for various schemes.

$\mathrm{rms}=\frac{1}{\overline{\bar{x}}}\left[\frac{\sum(x-\overline{\bar{x}})^{2}}{N}\right]^{1 / 2} \times 100 \%$,

$\max$ drift deviation $=\frac{\bar{x}_{\max }-\bar{x}_{\min }}{\overline{\bar{x}}} \times 100 \%$, where $T$ is the desired set-point target; $x$ is the value of an individual reading; $\overline{\bar{x}}$ is the grand average; $\bar{x}$ is the running average over 20 pulses; and $\bar{x}_{\max }$ and $\bar{x}_{\min }$ are the maximum and minimum values of $\bar{x}$ over the 4000 recorded pulses, respectively. 

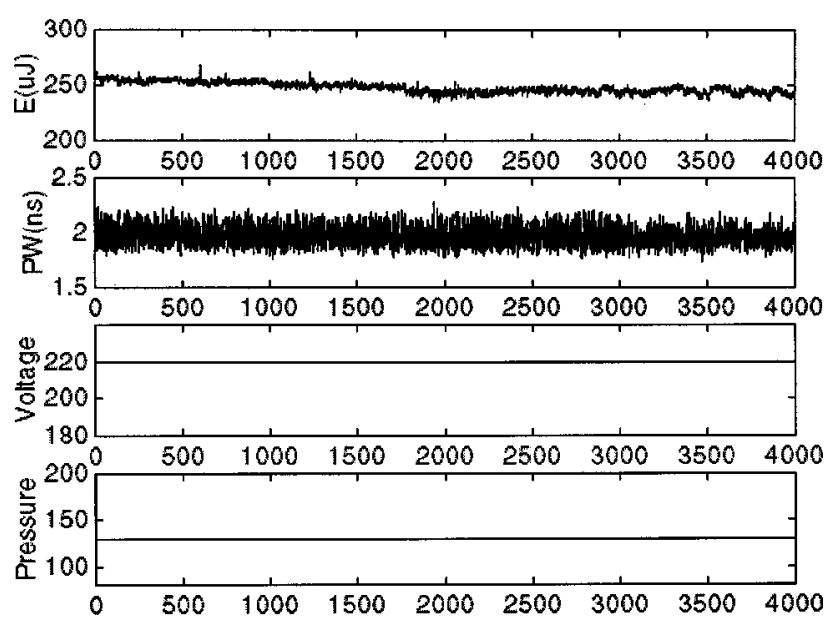

Fig. 9 Open-loop response.
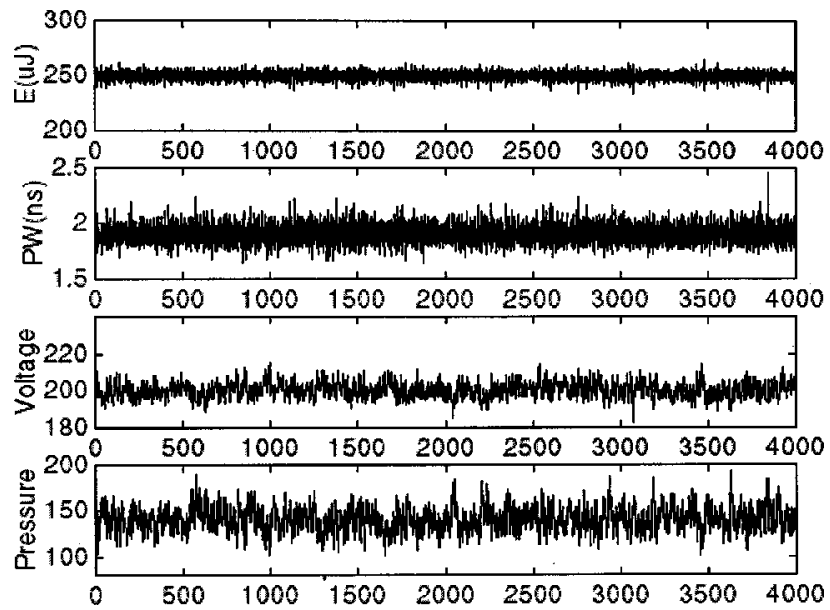

Fig. 10 Response using two-channel PID controller.

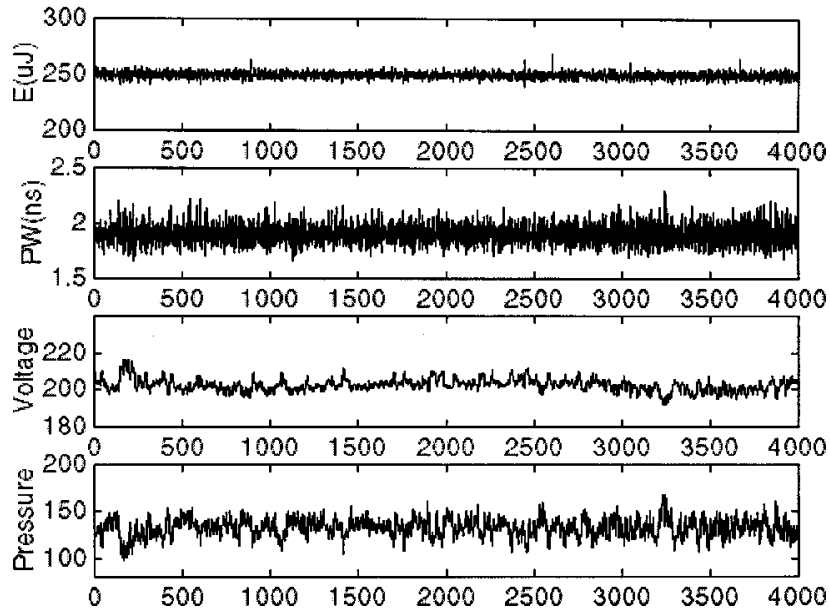

Fig. 11 Response using fuzzy logic controller.

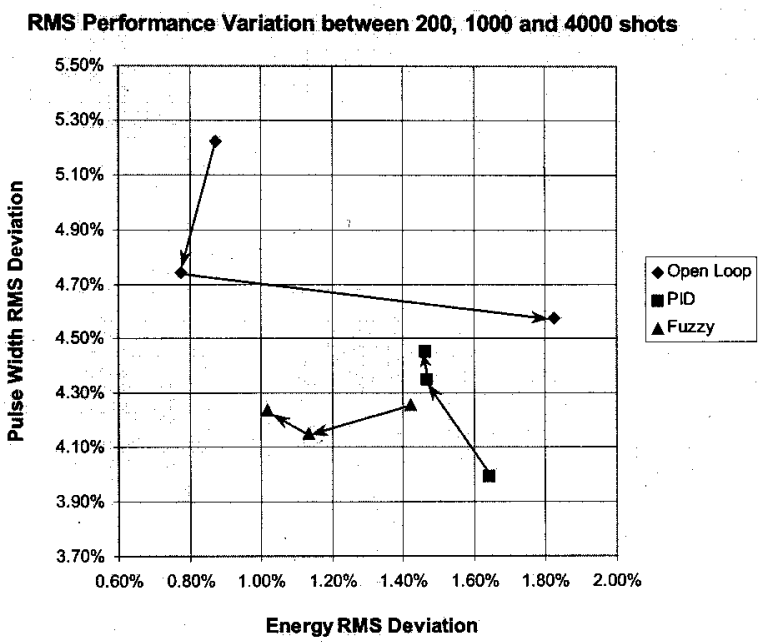

Fig. 12 The rms performance variation between 200, 1000 and 4000 shots.

Table 1 Summary of results $\left(E_{0}=0.25 \mathrm{~mJ}, \mathrm{PW}_{0}=1.90 \mathrm{~ns}\right)$.

\begin{tabular}{lccccccc}
\hline \hline & \multicolumn{3}{c}{ Energy $(\mu \mathrm{J})$} & & \multicolumn{2}{c}{ Pulse Width (ns) } \\
\cline { 2 - 4 } Control Scheme & Accuracy (\%) & rms Deviation (\%) & Max. Drift (\%) & & Accuracy (\%) & rms Deviation (\%) & Max. Drift (\%) \\
\hline Free-running laser & - & 1.82 & 7.03 & & - & 4.58 & 6.39 \\
PID control & 100.00 & 1.46 & 1.21 & & 100.00 & 4.46 & 3.03 \\
Fuzzy logic control (FL5) & 99.98 & 1.02 & 2.28 & & 99.92 & 4.24 & 3.88 \\
\hline \hline
\end{tabular}

The results of implementing the two-channel PID controller and the fuzzy logic controller are summarized in Table 1, and illustrated in Figs. 10 and 11 respectively. From Table 1, we observe that the two-channel PID controller performs better when compared to the openloop system. However, for every input setting, the user must fine-tune the control parameters extensively to achieve substantial output stability, and the same values of the control parameters cannot be reused once the input settings are changed. The fuzzy logic controller gives overall improve-ments when compared to the open-loop system. The rms instability for the pulse energy improves from 1.82 to $1.02 \%$, and that for the pulse width also improves from 4.58 to $4.24 \%$. The drift in pulse energy improves from 6.97 to $2.28 \%$ and that for the pulse width improves from 6.63 to $3.88 \%$. The changes in the system performances from 200 shots (short term) to 1000 shots (medium term) and finally to 4000 shots (long term) for the various schemes are shown in Fig. 12.

The performance of the fuzzy logic controller can be further enhanced if a learning scheme (also known as a 
supervisor or a critic) is introduced, e.g., using a neurofuzzy control algorithm. ${ }^{3}$

\section{Conclusions}

In this project, we have achieved the following results: First, closed-loop nitrogen laser was successfully constructed that operates within a voltage range of 9.0 to 11.1 $\mathrm{kV}$, and a pressure range of $250 \mathrm{mbar}$ to 1.0 bar. Second, closed-loop controllers were successfully implemented into the system. Feedback measurements on pulse energy and pulse width were used to fine-tune the operating discharge voltage and nitrogen pressure. Third, five different schemes of fuzzy logic control have been implemented and tested, and scheme FL5 gave the best results. Fourth, the results show that the PID controller and the fuzzy logic controller are able to track the outputs to the required targets very successfully. The performance of an FL controller was correlated to the surface plots. Finally, the fuzzy logic controller performed better than the PID controller in maintaining beam stability. Stability of the output pulse energy at $1.02 \%$ and that for the output pulse width at $4.24 \%$ were obtained simultaneously.

\section{Acknowledgments}

The authors would like to thank Mr. Song-Lip Lim and Mr. Kok-Tiong Goh for technical assistance, Dr. Duncan Campbell of La Trobe University, Australia, for technical discussions, and the Nanyang Technological University for funding this research project.

\section{References}

1. T. Kohonen, K. Makisara, O. Simula, and J. Kangas, Artificial Neural Networks, North-Holland (1991).

2. B. Kosko, Neural Networks and Fuzzy Systems, Prentice-Hall (1992).

3. C. T. Lin and C. S. G. Lee, Neural Fuzzy Systems: A Neuro-Fuzzy Synergism to Intelligence Systems, Prentice-Hall, Upper Saddle River, NJ (1996).

4. K. T. Goh and S. L. Lim, Neuro-Fuzzy Control of Nitrogen Laser, Final Year Report, School of EEE, NTU, Singapore (1998).

5. J. Nie and D. A. Linkens, Fuzzy-Neural Control: Principles, Algorithms, and Applications, Prentice-Hall (1995).

6. S. Lee, S. Kumar, T. C. Chia, A. Serban, and S. V. Springham, "Pulse charging of capacitors for reduction of voltage stress," Rev. Sci. Instrum. 65(7), 2408-2409 (1994).

7. S. C. Tam, W. P. Neo, H. C. Chua, A. T. S. Ho, and S. Lee, "A novel technique for measuring the pulsewidth of nanosecond laser pulses," IEEE Trans. Instrum. Meas. 47(4), 997-1000 (1998).

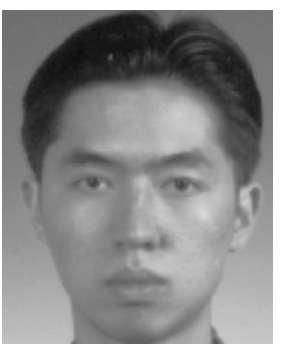

Siong-Chai Tan received his BEng degree in 1998 from the Nanyang Technological University, Singapore. He is currently pursuing his MEng degree in the same university. His present topic of research is intelligent control of nitrogen lasers.

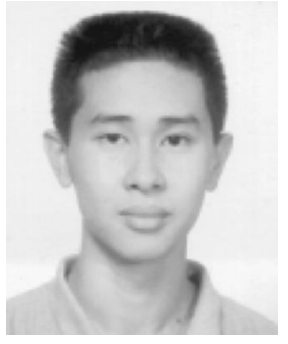

Sze-Chern Foong received his BEng degree (1st Class) in 1999 from the Nanyang Technological University, Singapore. He majored in control engineering and completed a final year project on intelligent control of lasers. He is currently working in DSO National Laboratories as a research and development engineer.

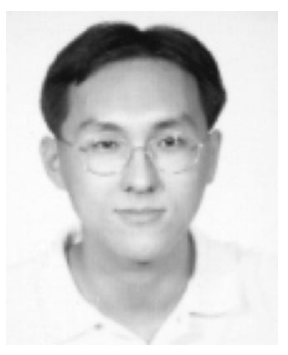

Choon-Hao Chan graduated from Nanyang Technological University in 1999 with a BEng (EEE) degree, specializing in computer engineering. He is presently working in National Computer Systems Pte. Ltd. as a systems engineer, focusing mainly on the operations support aspect for the Singapore Government Electronic Mail System project.

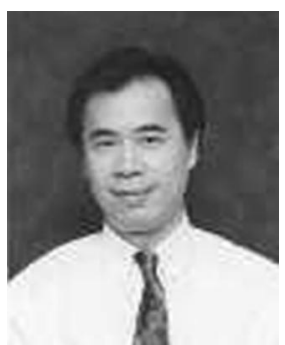

Anthony T. S. Ho received hi BSc (Honors) in physical electronics from the University of Northumbria, United Kingdom in 1979, and his MSc in applied optics from Imperial College, London, United Kingdom in 1980. He obtained his $\mathrm{PhD}$ in digital image processing (data compression) from King's College, University of London, United Kingdom in 1983. An engineer by training, Dr. Ho has worked in senior technical and management positions for various hi-tech software companies for more than 10 years in both United Kingdom and Canada. He joined Nanyang Technological University in 1994 and is currently an associate professor in the School of Electrical and Electronic Engineering. Dr. Ho is a fellow of the Institution of Electrical Engineers (IEE). He is also a senior member of IEEE, a chartered electrical engineer and a chartered physicist.

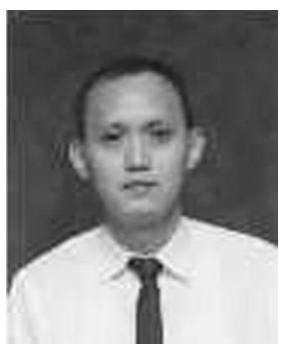

Hock-Chuan Chua is currently an associate professor in the School of Engineering, Nanyang Technological University. His research interest includes artificial intelligence and video processing.

Biographies of the other authors not available. 